\title{
A system for providing tactile EMG biofeedback
}

\author{
STEVEN L. SCHANDLER and WILLIAM W. GRINGS \\ University of Southern California, Los Angeles, California 90007
}

\begin{abstract}
This report describes a flexible and inexpensive biofeedback system for providing discrete pulses against the skin surface at a frequency proportional to the level of activity from selected muscle groups. The primary components consist of a voltage controlled pulse generator and a tactile transducer. Other system capabilities include the production of digital information for recording devices and the providing of pulsed auditory EMG biofeedback. The system may be particularly applicable for the experimental reduction of psychological and muscle tension.
\end{abstract}

EMG biofeedback has recently emerged as a reportedly effective technique for producing states of reduced tension in clinical and experimental situations (e.g., Basmajian, 1971; Budzynski \& Stoyva, 1973). Usually this procedure involves the use of apparatus supplying the subject with an auditory or visual analogue of selected muscle activity which the subject uses as an aid in reducing the level of that activity. However, the use of an auditory or visual presentation mode requires the subject to attend to an external source of information (speaker, meter, etc.) which may, therefore, inhibit the reduction of psychological tension.

The tactile EMG biofeedback system to be described was developed to present a less external information analogue than provided with either auditory or visual methods. The system functions to produce vibrations against the skin surface at a frequency directly related to the level of activity from selected muscles.

\section{DESCRIPTION}

A voltage controlled pulse generator and tactile transducer comprise the primary components of the tactile EMG biofeedback system.

\section{Voltage Controlled Pulse Generator}

The pulse generator is composed of an astable multivibrator and waveform/buffer amplifier. A circuit diagram of the system is provided in Figure 1.

Components R1, Q1, R2, IC1, C1, C2, and R3 form the multivibrator subsystem. R1 allows the selection of the portion of the EMG input signal to which the system will respond through the adjustment of current at the base of Q1. R2 serves as a limiting resistor through which IC1 draws current from Q1. ICl is physically a medium power dual two input gate configured to produce a square pulse of a duration adjustable by $\mathrm{R} 3$.

This research was supported by National Institute of Mental Health Research Grant MH-03916. Requests for reprints should be sent to William W. Grings, Department of Psychology, University of Southern California, Los Angeles, California 90007.

Steven L. Schandier is a predoctoral fellow supported by National Institute of Mental Health Grant MH-10554.
The waveform/buffer amplifier contains components Q2, R4, IC2, and R5. The output of IC1 is fed directly to amplifier Q2. IC2 is a medium power buffer receiving its input directly from Q2. The resulting pulse has an on amplitude of $+1 \mathrm{~V}$ and an off amplitude of $0 \mathrm{~V}$. Rise time is approximately 1 microsec.

\section{Tactile Transducer}

The transducer assembly is depicted in Figure 2. The transducer is $2-1 / 4$-in. diam, PM, 8 -ohm audio speaker. A backing consisting of $2-1 / 2 \times 2-1 / 2 \times 1 / 8$-in. foam pad and similar size phenolic board serves to protect the speaker cone and to attenuate any audio signals emitted by the transducer. An elastic strap is used to secure the transducer assembly to the subject's skin surface.

\section{OPERATION}

The system is designed to receive the output of EMG

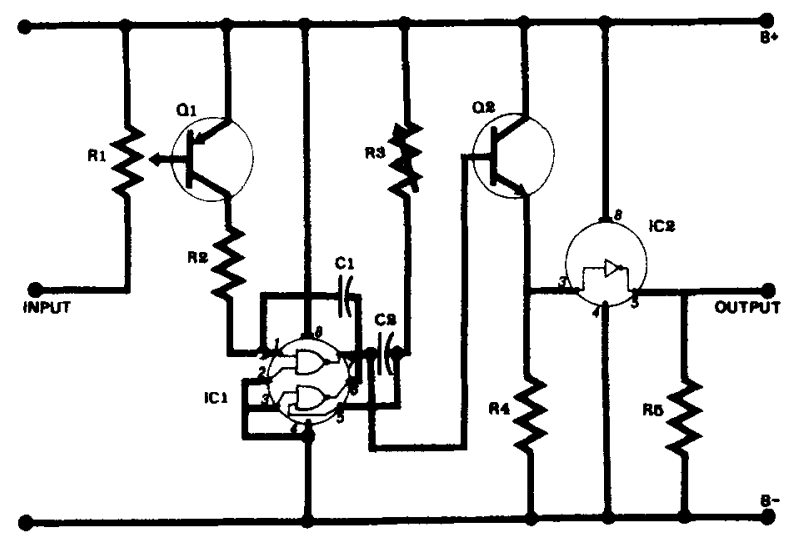

Figure 1. Circuit diagram of voltage controlled pulse generator. $B+=+6 \mathrm{~V}$ dc, $B=0 \mathrm{~V}$ dc. Parts List: $I C 1=$ Fairchild U8A991428X; IC2 = Fairchild U8A990028X; Q1 = Motorola 2N3638; Q2 = Motorola MPS6553; RI = Ohmite Type CB5031, $50 \mathrm{~K}$ ohm $; \mathrm{R2}=4 \mathrm{Kohm} ; \mathrm{C1}=8 \mathrm{microF} ; \mathrm{C2}=0.3 \mathrm{microF} ; \mathrm{R} 3=$ Ohmite Type CB2531, 25K ohm; R4, R5 = 1K ohm. 


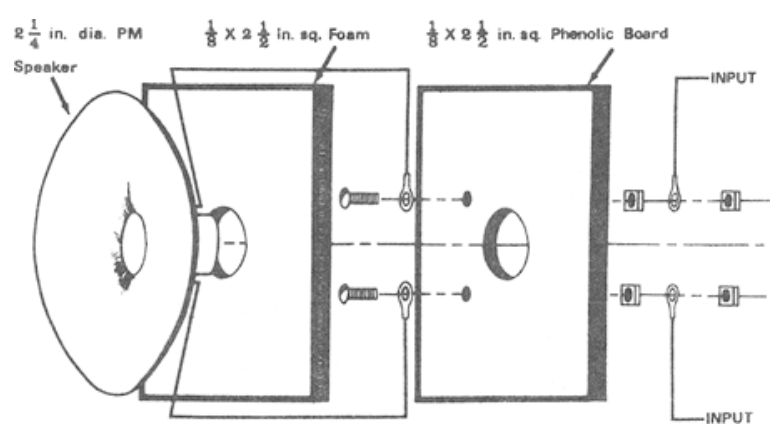

Figure 2. Exploded view of tactile transducer. The speaker press-fits into the phenolic board. An elastic strap is used to secure the unit to the skin surface.

recording devices delivering a signal of at least $.1 \mathrm{~V}$ p.p. During presentation of tactile EMG biofeedback, the output of the pulse generator is fed into an audio amplifier, which supplies the power to activate the tactile transducer. Increases in activity from the particular muscle group under examination will produce increases in the number of pulses presented to the subject's skin surface. Though the range of pulses is variable from none to $30 / \mathrm{sec}$, a range of 1 pulse every $3 \mathrm{sec}$ during low EMG activity to 10 pulses/sec during high EMG activity generally proves to be the most usable. The audio amplifier is used to adjust the force of the vibrations against the skin surface.

An external speaker or head phones can be substituted for the transducer to provide pulsed auditory EMG biofeedback. In addition, the output of the pulse generator can be used to drive digital recording devices.

\section{REFERENCES}

Basmajian, J. V. Conscious control and training of individual motor units and motor neurons. In J. Kamiya, et al. (Eds.), Biofeedback and self-control. Chicago: Aldine, 1971.

Budzynski, T. H., \& Stoyva, J. M. Biofeedback techniques in behavior therapy. In D. Shapiro, et al. (Eds.), Biofeedback and self-control. 1972, Chicago: Aldine, 1973.

(Received for publication August 6,1974, revision accepted October 1,1974 .) 